University of Wollongong

Research Online

Faculty of Engineering and Information

Faculty of Engineering and Information

Sciences - Papers: Part A

Sciences

$1-1-2013$

Dielectrophoretic manipulation and separation of particles in an S-shaped microchannel with hurdles

\author{
Ming Li \\ University Of Wollongong, ml433@uowmail.edu.au \\ Shunbo Li \\ Hong Kong University of Science and Technology \\ Weihua Li \\ University of Wollongong, weihuali@uow.edu.au \\ Weijia Wen \\ Hong Kong University of Science and Technology \\ Gursel Alici \\ University of Wollongong, gursel@uow.edu.au
}

Follow this and additional works at: https://ro.uow.edu.au/eispapers

Part of the Engineering Commons, and the Science and Technology Studies Commons 


\title{
Dielectrophoretic manipulation and separation of particles in an S-shaped microchannel with hurdles
}

\begin{abstract}
This paper presents a novel dielectrophoresis (DEP)-based microfluidic device, which incorporates multiple round hurdles within an S-shaped curved microchannel for continuous manipulation and separation of microparticles. Local nonuniform electric fields are induced by means of both constricted gaps formed between hurdles and outer channel wall, and variable current lengths in curved sections with equal width. Under the effect of negative DEP, particles will be directed away from either inner wall or hurdle edge, as they transport throughout the microchannel electrokinetically. Both experiment and numerical simulation were conducted, the results of which showed that fix-sized (i.e. 10 or $15 \mathrm{Pm}$ ) polystyrene (PS) particles could be successfully switched, directed and focused by adjusting applied voltages at inlet and outlets, and size-based separation of 10 and $15 \mathrm{Pm}$ particles was achieved with a careful selection of applied voltages. Compared to other microchannel designs that make use of either obstacle or curvature individually for inhomogeneous electric fields, this design offers advantages such as improved controllability over particle motion, lower requirement of applied voltage, reduced fouling and particle adhesion, etc.
\end{abstract}

\section{Keywords}

shaped, microchannel, hurdles, dielectrophoretic, manipulation, particles, separation

\section{Disciplines}

Engineering | Science and Technology Studies

\section{Publication Details}

Li, M., Li, S., Li, W., Wen, W. \& Alici, G. (2013). Dielectrophoretic manipulation and separation of particles in an S-shaped microchannel with hurdles. 2013 IEEE/ASME International Conference on Advanced Intelligent Mechatronics (AIM) (pp. 362-366). United States: IEEE. 


\title{
Dielectrophoretic manipulation and separation of particles in an S-shaped microchannel with hurdles*
}

\author{
Ming Li, Shunbo Li, Weihua Li, Weijia Wen, and Gursel Alici
}

\begin{abstract}
This paper presents a novel dielectrophoresis (DEP)-based microfluidic device, which incorporates multiple round hurdles within an S-shaped curved microchannel for continuous manipulation and separation of microparticles. Local nonuniform electric fields are induced by means of both constricted gaps formed between hurdles and outer channel wall, and variable current lengths in curved sections with equal width. Under the effect of negative DEP, particles will be directed away from either inner wall or hurdle edge, as they transport throughout the microchannel electrokinetically. Both experiment and numerical simulation were conducted, the results of which showed that fix-sized (i.e. 10 or $15 \mu \mathrm{m}$ ) polystyrene (PS) particles could be successfully switched, directed and focused by adjusting applied voltages at inlet and outlets, and size-based separation of 10 and $15 \mu \mathrm{m}$ particles was achieved with a careful selection of applied voltages. Compared to other microchannel designs that make use of either obstacle or curvature individually for inhomogeneous electric fields, this design offers advantages such as improved controllability over particle motion, lower requirement of applied voltage, reduced fouling and particle adhesion, etc.
\end{abstract}

\section{INTRODUCTION}

Dielectrophoresis (DEP), first adopted by Pohl [1], is a phenomenon that occurs due to a translational force exerted on a dielectric particle in a nonuniform electric field. With the rapid development of lab-on-a-chip (LOC) devices in recent years, it has been widely used as one of the most popular methods to manipulate various micro/nano scale bioparticles (i.e. DNA, protein, bacteria, virus, mammalian and yeast cells) in microfluidic systems [2-3]. Compared to other (i.e. mechanical, thermal, magnetic, acoustic, optical, chemical and electrical) techniques applied within microfluidics [4], DEP offers a number of significant advantages: label-free nature, favourable scaling effects, simplicity of the instrumentation, ability to manipulate neutral bioparticles, and analysis of high selectivity and sensitivity [3, 5-6].

Traditionally, the spatial nonuniformities required for DEP effect are generated by applying alternative current (AC) electric fields to the microelectrodes patterned within microchannels [3, 7], however, such electrode-based DEP microdevices suffer from fabrication complexities due to electrode construction, electrode fouling [8], and

*Research supported by University of Wollongong through an international links project.

M. Li, W. Li, and G. Alici are with School of Mechanical, Materials and Mechatronic Engineering, University of Wollongong, Wollongong, NSW 2522, Australia (corresponding author: Prof. Weihua Li; phone: +61 24221 3490; fax: +61 24221 3238; e-mail: weihuali@uow.edu.au).

S. Li and W. Wen are with Department of Physics, The Hong Kong University of Science of Technology, Clear Water Bay, Kowloon, Hong Kong (e-mail: phwen@ust. hk). electrochemical reactions on the electrode surface [9]. These problems are avoided in insulator-based DEP microdevices, where direct current (DC) or DC-biased AC electric fields are applied via external electrodes submerged in inlet and outlet reservoirs, and electric field gradients are induced around insulating objects. In such devices, two main approaches have been used to generate required nonuniform electric fields [10-11]: obstacles and microchannel curvature. However, microdevices with electrically insulated obstacles (i.e. including posts, rectangular/triangular hurdles, ridge, oil droplet, and oil menisci) embedded in straight microchannels, have such limitations as locally amplified electric fields, large trans-membrane voltages and shear stresses on cells, Joule heating, and fouling due to particle clogging or adhesion [12]. Although the highly-intensify local electric fields can be avoided in curved insulating microchannels, including sawtooth, serpentine, circular, spiral, waved microchannels, this curvature-based method requires sufficiently large applied DC voltage and/or long curved section for effective performance of the device, inducing complexities in terms of operation and fabrication. In addition, the device is more sensitive to contamination (i.e. particle adhesion on channel wall), as long channel increases the possibility of surface inhomogeneity.

In this work, we developed a design combing the effects of obstacle and curvature to generate electric field gradient required for the DEP effect, where multiple round hurdles are embedded within an S-shaped curved microchannel to achieve continuous particle manipulation and separation. The aforementioned adverse effects of using each approach individually, such as locally applied electric fields, particle clogging, large applied voltage, etc., have been significantly reduced. Moreover, the desired functionality of the microfluidic device can be achieved more easily due to the increase of approach for particle manipulation. The manipulation functioning of the design was demonstrated by controlling the trajectories of 10 or $15 \mu \mathrm{m}$ PS particles by adjusting applied voltages. In addition, the separation functioning was verified by continuously separating 10 and $15 \mu \mathrm{m}$ PS particles according to their differences in size. Both experimental and numerical results were presented, which showed a reasonable agreement.

\section{THEORY}

Particles suspended in an electrically conducting liquid under the influence of external electric field are subjected to electrophoretic, electroosmotic and dielectrophoretic effect. The combination of fluid electroosmosis (EO) and particle electrophoresis (EP) is termed electrophoretic (EK) flow, resulting in the electrokinetic velocity of particles written as [13] 


$$
\mathbf{u}_{E K}=\mu_{E K} \mathbf{E}=\mathbf{u}_{E O}+\mathbf{u}_{E P}=\left(\mu_{E O}-\mu_{E P}\right) \mathbf{E}
$$

where $\mu_{E K}, \mu_{E O}=-\varepsilon_{m} \zeta_{w} / \eta$ and $\mu_{E P}=-\varepsilon_{m} \zeta_{p} / \eta$ are electrokinetic, electroosmotic, and electrophoretic mobility, respectively. $\varepsilon_{m}$ and $\eta$ are the permittivity and dynamic viscosity of the suspending medium, respectively. $\zeta_{w}$ and $\zeta_{p}$ represent, respectively, the zeta potential of the channel and the particle. $\mathbf{E}$ is the electric field vector. Equation (1) shows that the electrokinetic velocity of the particle is linearly proportional to the local electric field, leading to the particle transport along the electric lines.

Using the dipole moment method, the time-average DEP force and the induced dielectrophoretic velocity acting on a dielectric spherical particle in a nonuniform DC electric field are given by [14]

$$
\mathbf{F}_{D E P}=(1 / 2) \pi \varepsilon_{m} d^{3} f_{C M}(\mathbf{E} \cdot \nabla \mathbf{E})
$$

and

$$
\mathbf{u}_{D E P}=\mu_{D E P}(\mathbf{E} \cdot \nabla \mathbf{E})=\left(\varepsilon_{m} d^{2} f_{C M} / 6 \eta\right) \cdot(\mathbf{E} \cdot \nabla \mathbf{E})
$$

where $d$ is the particle diameter, $\mu_{D E P}$ is the dielectrophoretic mobility, $f_{C M}=\left(\sigma_{p}-\sigma_{m}\right) /\left(\sigma_{p}+2 \sigma_{m}\right)$ is known as the Clausius-Mossotti (CM) factor, $\sigma_{p}$ and $\sigma_{m}$ are the electric conductivities of particle and the suspending medium, respectively. If the particle is less conductive than the suspending medium $\left(\sigma_{p}<\sigma_{m}\right)$, CM factor will be negative $\left(f_{C M}<0\right)$, inducing a negative DEP force that repels particles away from the region of higher electric field.

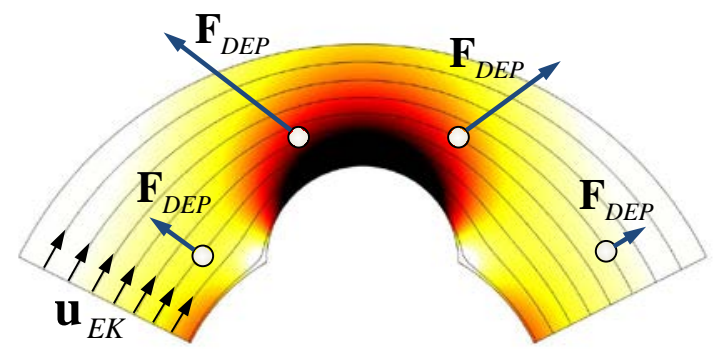

Figure 1. Illustration of the negative dielectrophoretic maniupulation and separation of particles in a curved microchannel embedded with a round hurdle. Distribution of the electric-field lines and contours of the electric-field strength $(\mathrm{E})$ within the microchannel are shown (darker region has a stronger electric field). Particles moving through the microchannel electrokinetically are subjected to negative DEP forces (indicated by dark blue arrows).

Herein, we utilized the effect of both obstacle and curvature to generate local electric field gradient throughout the microchannel, which contributes to a novel technique for the continuous control of particle movement in a microfluidic chip by DEP effect. The mechanism of the proposed design is illustrated schematically in Figure (1), where a semi-circular microchannel embedded with a round hurdle are presented along with the electric field lines (or equivalently the streamlines with black arrows indicating the direction) and contours of the electric field strength (the darker the stronger). The overall distribution of electric field is as follows: a relatively weaker and slightly nonuniform electric field is generated in the width direction of the curved channel with uniform cross sections; whereas, a stronger and highly nonuniform electric field is created near the edge of the hurdle (electric field obtained maximum value at the hurdle edge and decayed as increasing distance away from the hurdle). Consider a particle subjected to negative DEP effect passing though the microchannel under the combined effect of EOF and EP, repulsive DEP forces (dark blue arrows, relatively weak in the curved section, while strong in the constricted region) are exerted on the particle all along its movement.

\section{MATERIALS AND METHODS}

\section{A. Microchannel Fabrication and Layout}

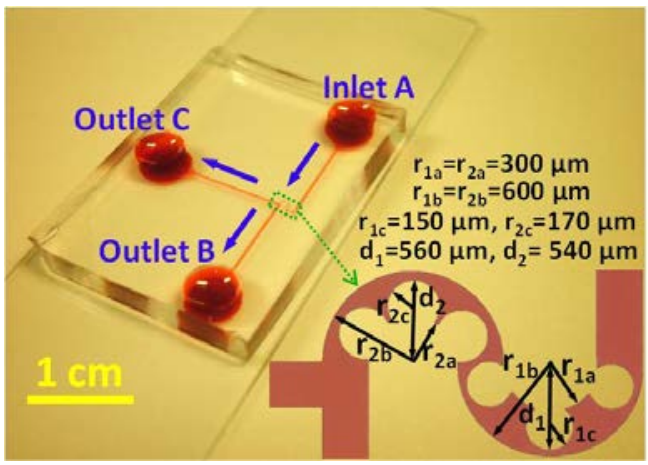

Figure 2. Photograph of the DEP-based microfluidic chip for continuous particle manipulation and separation. The inset indicates the structrues and dimensions of the design.

The polydimethylsiloxane (PDMS) microfluidic channel was fabricated using standard soft-lithography technique, and a detailed fabrication process can be found in our previous work [15]. As shown in Figure (2), the microfluidic chip is composed of two semi-circular channels that each integrates with three round hurdles from inner wall, one inlet $(A)$ and two outlet (B and C) reservoirs, and three straight connecting microchannels. Both semi-circular channels are $300 \mu \mathrm{m}$ in width, having a large curvature of $600 \mu \mathrm{m}$, while a small curvature of $300 \mu \mathrm{m}$. The round hurdles in the first and second curved channel have radius of, respectively, $150 \mu \mathrm{m}$ and $170 \mu \mathrm{m}$, creating $40 \mu \mathrm{m}$ and $60 \mu \mathrm{m}$-wide gaps between hurdle and outer wall, respectively. All three straight connecting channels have a width of $300 \mu \mathrm{m}$ and a length of 1 $\mathrm{mm}$. The entire microchannel has the same depth of $40 \mu \mathrm{m}$. All three reservoirs at the inlet and outlets are $3 \mathrm{~mm}$ in height and $6 \mathrm{~mm}$ in diameter. 


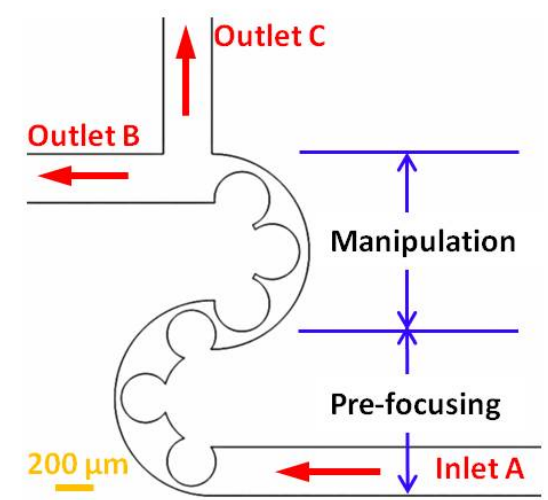

Figure 3. Schematic diagram of the S-shaped microchannel embedded with multiple round hurdles.

As indicated in Figure (3), the proposed microchannel taking advantage of curvature and hurdle for continuous particle manipulation and separation consists of two units. The first (or clockwise) unit connects with an inlet, while the second (or counterclockwise) unit connects with two outlets placing with an intersection angle of 90 degrees. Both units incorporate a semi-circular microchannel with three round hurdles from the inner wall. Multiple round hurdles were chosen to enhance DEP effect and improve device performance for particle manipulation and separation [16]. Moreover, it has been stated in the previous work of Kang et al [17] that incoming position of particles could affect the particle trajectory shift after passing the block, hence the first curved section of hurdle-embedded semi-circular microchannel for pre-processing was designed. The first and second curved section are for particle pre-focusing and post-manipulation (or separation), respectively. More importantly, the first curved channel with embedded round hurdles was designed to pre-focus particles close to the hurdle region within the second curved unit for subsequent manipulation, therefore, it allows for increased efficiency of switching and sorting particles.

\section{B. Sample Preparation}

In this study, two types of particles with varied sizes (Fluospheres, Invotrogen, CA, USA) were utilized: yellow-green fluorescent $10 \mu \mathrm{m}$ and blue fluorescent $15 \mu \mathrm{m}$ polystyrene (PS) microspheres. Both original particle solutions were diluted by deionized (DI) water 15 times. For separation experiment, the diluted 10 and $15 \mu \mathrm{m}$ particle solutions were mixed at a volume ratio of $1: 1$. The particle solutions were gently vibrated prior to be introduced into the inlet reservoir using a pipette. Moreover, the outlet reservoirs were filled with the corresponding working solution, and the liquid level in each reservoir was carefully balanced before applying DC voltages via submerged platinum (Pt) electrodes.

\section{Experimental Setup}

The electric field was generated by a DC power supply (SL10P300/200, Spellman High Voltage Electronics Corp., Hauppauge, NY). The motion of particle through the microchannel was monitored and recorded by an inverted microscope (Olympus IX71, Tokyo, Japan) equipped with a CCD camera (DP 70, Olympus, Tokyo, Japan). The camera was run in the video mode at the speed of 15 frames per second and the acquired digital images had a resolution of 680 X 512 pixels. All the videos and images were post-processed by MATLAB (Mathworks Inc., Natick, MA), and the particle trajectories were obtained by superimposing consecutive images converted from videos.

\section{Numerical Modeling}

In the simulation, we used a two-dimensional (2D) model that first developed by Kang et al [18], and has also validated by other researchers in various microfluidic channel structures [15, 19-21] to predict the particle motion throughout the microchannel. By introducing a correction factor, $C$, to account for the effects of particle size, particle-particle interaction, etc. on the dielectrophoretic velocity, the velocity of particle can be written as

$$
\mathbf{u}_{p}=\mathbf{u}_{E K}+c \mathbf{u}_{D E P}=\mu_{E K} \mathbf{E}+c \mu_{D E P}(\mathbf{E} \cdot \nabla \mathbf{E})
$$

The above equation was performed in COMSOL 4.0 to predict the particle trajectory, where the electrokinetic mobility and the dielectrophoretic mobility were calculated by Equation (1) and Equation (3), respectively. The zeta potential values of PS particles and channel wall in $10 \mathrm{mM}$ $\mathrm{NaCl}$ solution were set to be -33 and $-54 \mathrm{mV}$, respectively [22-23] . The dynamic viscosity, $\eta=0.9 \times 10^{-3} \mathrm{~kg} /(\mathrm{m} \cdot \mathrm{s})$, and permittivity, $\varepsilon_{m}=6.9 \times 10^{-10} \mathrm{C} /(\mathrm{v} \cdot \mathrm{m})$, for pure water at $25^{\circ} \mathrm{C}$ were also used. As the electric conductivity of polystyrene particle in DC electric field is much smaller than that of suspending medium (i.e. $10 \mathrm{mM} \mathrm{NaCl}$ solution) used in our experiments, the CM factor, $f_{C M}$, which depends on electrical conductivities of both the particle and the suspending medium was set to be -0.5 .

\section{RESUltS AND DisCUSSION}

A. Continuous Manipulation of Particles

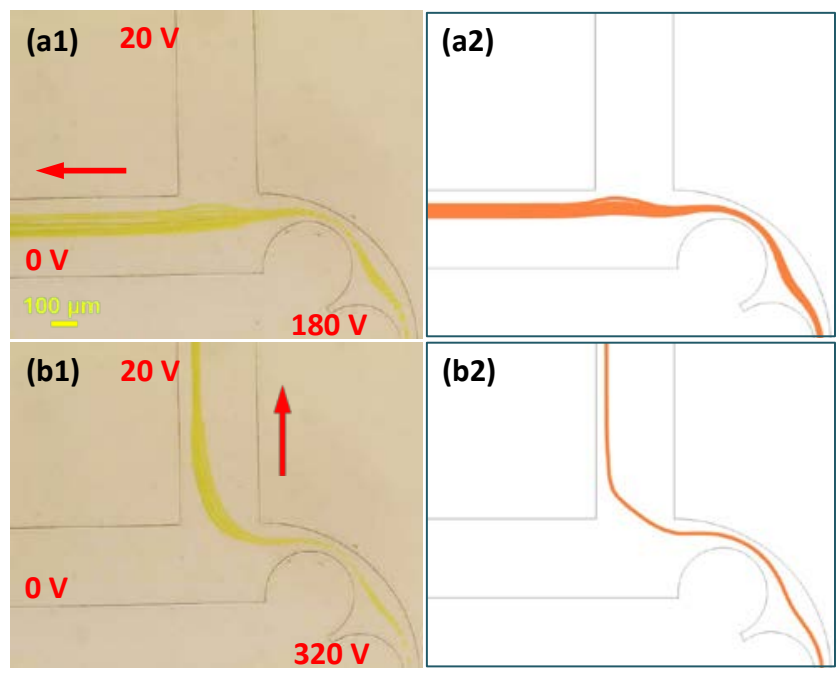

Figure 4. Experimental (left column: superimposed images) and numerical (right column) demonstration of manipulating $10 \mu \mathrm{m}$ particles. The applied voltage at the inlet was increased from (a) $180 \mathrm{~V}$ to (b) $320 \mathrm{~V}$, while applied voltages at outlet $B$ and outlet $C$ were fixed at 0 and $20 \mathrm{~V}$, respectively. The red arrows indicate the flow direction. 
Figure (4) shows the comparison between experimentally obtained (left column) and numerically predicted (right column) results of $10 \mu \mathrm{m}$ particle trajectories under varied inlet voltages. It can be found that at lower inlet voltage (a: $180 \mathrm{~V}$ ), all particles were moved out of the microchannel from outlet $\mathrm{B}$, however, particles were directed to a narrower stream in outlet $\mathrm{C}$ at higher applied voltage (b: $320 \mathrm{~V}$ ). According to Equation (2), DEP force depends on the gradient of the square of the electric field, larger applied voltage can induce larger repulsive DEP effect, which in turn causes particles to be deflected further away from the hurdle and inner wall. The right column of Figure (4) represents the numerically predicted $10 \mu \mathrm{m}$ particle trajectories in the same conditions as those in the experiments. The correction factor was set to be 0.5 by matching the simulated results to those of obtained superimposed images, which remained constant for particles of fixed size in all the cases.

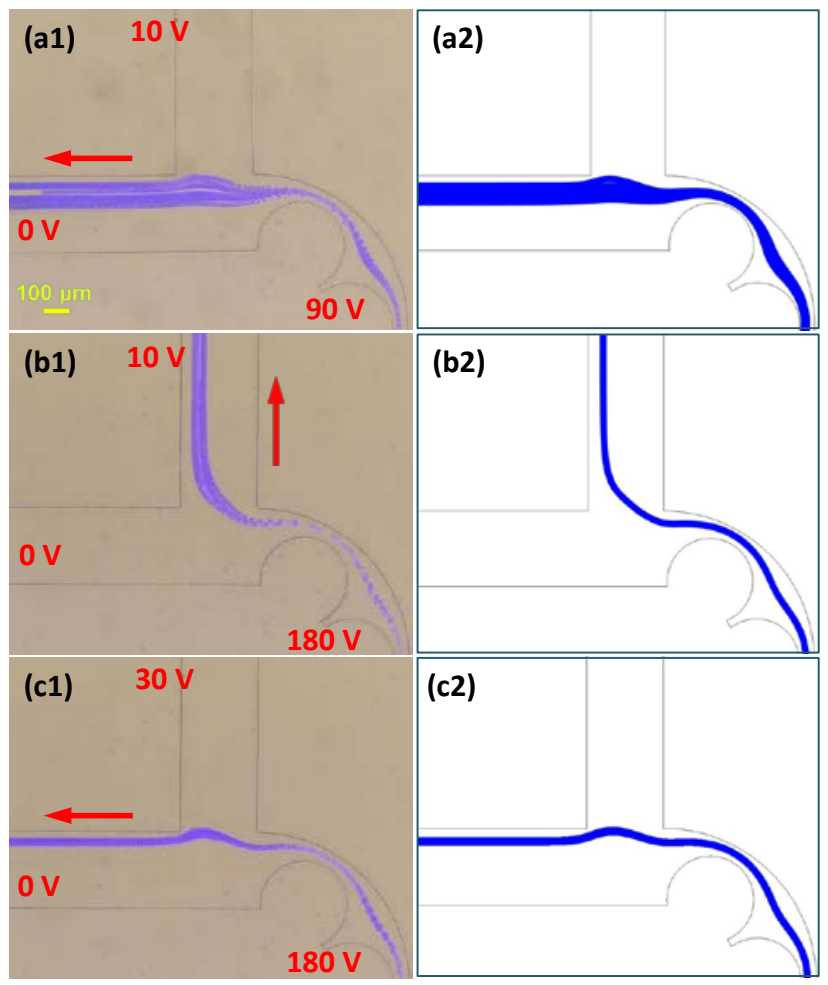

Figure 5. Experimental (left column: superimposed images) and numerical (right column) demonstration of manipulating $15 \mu \mathrm{m}$ particles. The applied voltage to inlet and outlet C were, respectively: (a) 90 and $10 \mathrm{~V}$; (b) 180 and

$10 \mathrm{~V}$; (c) 180 and $30 \mathrm{~V}$, while the outlet B was grounded in all cases.

In similar analysis, we studied experimentally and numerically how to control the motion of $15 \mu \mathrm{m}$ particles by adjusting applied voltages at inlet and outlet. Figure (5) represents the experimentally observed (left column: superimposed images) trajectories of $15 \mu \mathrm{m}$ particles at the exit region of the microchannel under different combinations of inlet and outlet voltages: (a) when applied voltages at inlet, outlet $\mathrm{B}$ and outlet $\mathrm{C}$ were, respectively, 90,0 and $10 \mathrm{~V}$, particles moved out of the microchannel in a confined stream from outlet B; (b) by increasing the inlet voltage to $180 \mathrm{~V}$ but fixing outlet voltages, particles were pushed further from round hurdles and directed to outlet $\mathrm{C}$ in a narrower stream due to larger repulsive DEP force; (c) further increased the outlet C voltage to $30 \mathrm{~V}$, but fixed outlet B to be grounded, particles were diverted to move from outlet $\mathrm{B}$ again due to the re-distribution of the flow at the bifurcation. It could be found that applied voltages at both inlet and outlet could affect particle trajectory, and $15 \mu \mathrm{m}$ particles can be directed into either outlet $\mathrm{B}$ or outlet $\mathrm{C}$ depending on the applied voltages. In addition, with the increase of inlet and/or outlet voltage, 15 $\mu \mathrm{m}$ particles were observed to obtain a better focusing effect (forced into a narrower stream), which corresponds to our previous founding that the performance of particle focusing could be improved with the rise of applied electric field [15]. By setting the correction factor to be 0.4 for $15 \mu \mathrm{m}$ particles, the numerically predicted results (right column) coincides acceptably with the experimentally observed superimposed images (left column).

\section{B. Continuous Size-based Separation of Particles}

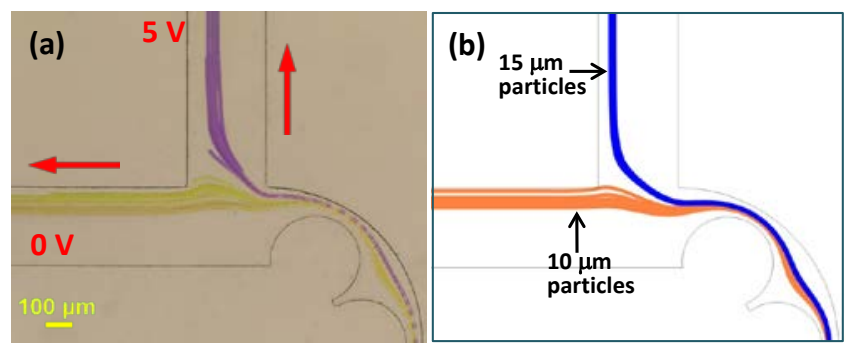

Figure 6. Continuous separation of 10 (yellow) and $15 \mu \mathrm{m}$ (blue) particles in the proposed design, when the applied voltages at inlet $A$ and outlet $C$ were 160 and $5 \mathrm{~V}$, respectively, and outlet B was grounded: (a) experimentally obtained superimposed image, and (b) numerically predicted result. The red arrows indicate the flow direction.

According to Equation (2), the DEP force is proportional to the cube of particle diameter, therefore, larger particles are subjected to larger DEP forces, and hence tend to be deflected further from the hurdle edge and inner wall compared to smaller ones. This mechanism is utilized in the proposed microchannel to perform continuous separation of particles based on size. A typical case of the separation of mixed 10 (yellow) and $15 \mu \mathrm{m}$ (blue) PS particles with applied voltages at inlet, outlet B and outlet $\mathrm{C}$ of 160, 0 and $5 \mathrm{~V}$, respectively, is shown in Figure (6). It can be seen that there were two distinct particle streams at the bifurcation, as 10 and $15 \mu \mathrm{m}$ particles were sorted and moved in a focused stream from outlet B and outlet C, respectively. The separation performance was also reasonably predicted by numerical simulation (see right column of Figure 6), in which the trajectories of 10 and $15 \mu \mathrm{m}$ particles were represented by yellow and blue lines, respectively, and the correction factors were fixed to be 0.4 and 0.5 , respectively, for 10 and $15 \mu \mathrm{m}$ particles.

\section{CONCLUSION}

In this work, an S-shaped curved microchannel embedded with multiple round hurdles for continuous manipulation and separation of microparticles using negative DEP was presented. In such design, the effect of obstacle and curvature are combined to induce nonuniform electric fields, generating controllable local electric fields all along the microchannel. Both experimental and numerical results indicated that by adjusting applied voltages at the inlet and outlets, 
microparticles that can be directed into distinct outlets, positioned at different locations along the outlet width direction, and focused into streams of varied widths. Moreover, size-dependent separation was achieved for sorting 10 and $15 \mu \mathrm{m}$ PS particles simultaneously. In general, a good agreement for the particles trajectories is found between the experimental observation and the numerical prediction.

The proposed hurdle-combined curved microchannel has great advantages: (1) it provides more parameters (i.e. gap width, configuration of hurdle and curved channel) to be optimized, leading to increased controllability of the particle motion; (2) it relives or eliminates the problems that occur in the insulator-based DEP microdevices that taking advantage of either obstacle or curvature effect individually for electric field gradients, allowing the improvement of device performance; (3) the upstream curved unit, a semi-circular microchannel combined with round hurdle, was designed to pre-focus particles into a stream close to the hurdle region, thus facilitates subsequent process of particle manipulation and separation. The novel design with demonstrated integrated functionality of focusing, switching and sorting microparticles is expected to be widely used in LOC devices for biological, chemical and medical applications.

\section{ACKNOWLEDGMENT}

The author (Miss Ming Li) wishes to thank the China Scholarship Council and the University of Wollongong for offering joint $\mathrm{PhD}$ scholarships.

\section{REFERENCES}

[1] Pohl, H.A., Dielectrophoresis : the behavior of neutral matter in nonuniform electric fields. 1978, Cambridge: Cambridge University Press.

[2] Pethig, R., Review Article-Dielectrophoresis: Status of the theory, technology, and applications. Biomicrofluidics, 2010. 4(2).

[3] Zhang, C., et al., Dielectrophoresis for manipulation of micro/nano particles in microfluidic systems. Analytical and Bioanalytical Chemistry, 2010. 396(1): p. 401-420.

[4] Gossett, D.R., et al., Label-free cell separation and sorting in microfluidic systems. Analytical and Bioanalytical Chemistry, 2010. 397(8): p. 3249-3267.

[5] Gascoyne, P.R.C. and J. Vykoukal, Particle separation by dielectrophoresis. Electrophoresis, 2002. 23(13): p. 1973-1983.

[6] Hughes, M.P., Strategies for dielectrophoretic separation in laboratory-on-a-chip systems. Electrophoresis, 2002. 23(16): p. 2569-2582.

[7] Tay, F.E.H., L. Yu, and C. Iliescu, Particle Manipulation by Miniaturised Dielectrophoretic Devices. Defence Science Journal, 2009. 59(6): p. 595-604.

[8] Voldman, J., Electrical forces for microscale cell manipulation, in Annual Review of Biomedical Engineering. 2006. p. 425-454.

[9] Gencoglu, A. and A. Minerick, Chemical and morphological changes on platinum microelectrode surfaces in AC and DC fields with biological buffer solutions. Lab on a Chip, 2009. 9(13): p. 1866-1873.

[10] Regtmeier, J., et al., Electrodeless dielectrophoresis for bioanalysis: Theory, devices and applications. Electrophoresis, 2011. 32(17): p. 2253-2273.

[11] Srivastava, S.K., A. Gencoglu, and A.R. Minerick, DC insulator dielectrophoretic applications in microdevice technology: a review. Analytical and Bioanalytical Chemistry, 2011. 399(1): p. 301-321.

[12] Xuan, X., J. Zhu, and C. Church, Particle focusing in microfluidic devices. Microfluidics and Nanofluidics, 2010. 9(1): p. 1-16.
[13] Ramos, A., et al., Ac electrokinetics: a review of forces in microelectrode structures. Journal of Physics D-Applied Physics, 1998. 31(18): p. 2338-2353.

[14] Jones, T.B., Electromechanics of Particles. 1995, Cambridge: Cambridge Unviersity Press.

[15] Li, M., et al., Continuous particle focusing in a waved microchannel using negative dc dielectrophoresis. Journal of Micromechanics and Microengineering, 2012. 22(9).

[16] Lewpiriyawong, N., C. Yang, and Y.C. Lam, Dielectrophoretic manipulation of particles in a modified microfluidic $H$ filter with multi-insulating blocks. Biomicrofluidics, 2008. 2(3).

[17] Kang, K.H., et al., Effects of dc-dielectrophoretic force on particle trajectories in microchannels. Journal of Applied Physics, 2006. 99(6).

[18] Kang, K.H., et al., Continuous separation of microparticies by size with direct current-dielectrophoresis. Electrophoresis, 2006. 27(3): p. 694-702.

[19] Zhu, J., et al., DC dielectrophoretic focusing of particles in a serpentine microchannel. Microfluidics and Nanofluidics, 2009. 7(6): p. 751-756.

[20] Zhu, J. and X. Xuan, Particle electrophoresis and dielectrophoresis in curved microchannels. Journal of Colloid and Interface Science, 2009. 340(2): p. 285-290.

[21] Zhu, J. and X. Xuan, Dielectrophoretic focusing of particles in a microchannel constriction using DC-biased AC flectric fields. Electrophoresis, 2009. 30(15): p. 2668-2675.

[22] Takahashi, N., et al., Proposal and experimental validation of the electrophoretic Coulter method for analyzing microparticles and biological cells. Sensors and Actuators B-Chemical, 2011. 151(2): p. 410-415.

[23] Venditti, R., X. Xuan, and D. Li, Experimental characterization of the temperature dependence of zeta potential and its effect on electroosmotic flow velocity in microchannels. Microfluidics and Nanofluidics, 2006. 2(6): p. 493-499. 